\title{
In vitro and in vivo magnetic resonance imaging with chlorotoxin-conjugated superparamagnetic nanoprobes for targeting hepatocarcinoma
}

\author{
ZHU CHEN $^{1}$, EN-HUA XIAO ${ }^{1}$, ZHEN KANG ${ }^{1}$, WEN-BIN ZENG ${ }^{2}$, HUI-LONG TAN ${ }^{2}$, \\ HUA-BING LI ${ }^{1}$, DU-JUN BIAN ${ }^{1}$ and QUAN-LIANG SHANG ${ }^{1}$ \\ ${ }^{1}$ Department of Radiology, Second Xiangya Hospital, Central South University, Changsha, Hunan 410011; \\ ${ }^{2}$ School of Pharmaceutical Sciences, Central South University, Changsha, Hunan 410013, P.R. China
}

Received December 15, 2015; Accepted January 21, 2016

DOI: $10.3892 /$ or.2016.4629

\begin{abstract}
The present study aimed to assess the in vitro and in vivo magnetic resonance imaging (MRI) features of chlorotoxin (CTX)-conjugated superparamagnetic iron oxide (SPIO) nanoprobes. CTX-conjugated nanoprobes were composed of SPIO coated with polyethylene glycol (PEG) and conjugated with CTX. The nanoprobes were termed SPIO-PEG-CTX. MRI of the SPIO and SPIO-PEG-CTX solutions at a different concentration was performed with a 3.0-T MRI scanner (Philips Achieva 3.0T X Series; Phillips Healthcare, The Netherlands). Rabbit VX2 hepatocarcinoma was established by a traditional laparotomy method (injection of the tumor particles into the liver using a $15 \mathrm{G}$ syringe needle) following approval by the institutional animal care and use committee. Contrast-enhanced MRI of VX2 rabbits $(n=8)$ was performed using the same MRI scanner with SPIO-PEG-CTX solutions as the contrast agent. Data were analyzed with calibration curve and a paired t-test. The SPIO-PEG-CTX nanoparticles were successfully prepared. With increasing concentrations of the solutions, the MRI signal intensity was increased at T1WI, but decreased at T2WI, which were the same as that for SPIO. Rabbit VX2 carcinoma appeared as a low MRI signal at T1WI, and high at T2WI. After injection of the contrast agent, the MRI signal of carcinoma was decreased relative to that before injection at T2WI $(1,161 \pm 331.5$ vs. $1,346 \pm 300.5$; $\mathrm{P}=0.004<0.05$ ), while the signal of the adjacent normal hepatic tissues was unchanged $(480.6 \pm 165.1$ vs. $563.4 \pm 67.8$; $\mathrm{P}=0.202>0.05)$. The SPIO-PEG-CTX nanoparticles showed MRI negative enhancement at T2WI and a targeting effect in
\end{abstract}

Correspondence to: Professor En-Hua Xiao, Department of Radiology, Second Xiangya Hospital, Central South University, 139 Renmin Middle Road, Changsha, Hunan 410011, P.R. China E-mail: cjr.xiaoenhua@vip.163.com

Key words: magnetic resonance imaging, superparamagnetic iron oxide, chlorotoxin, in vitro, in vivo, hepatocarcinoma liver cancer, which provides the theoretical basis for further study of the early diagnosis of hepatocellular carcinoma.

\section{Introduction}

Liver cancer, the third leading cause of cancer-related death worldwide, is a primary malignant cancer originating in the liver. There are $\sim 60-70$ million liver cancer-related deaths each year, $50 \%$ of which occur in China. Most patients are diagnosed at intermediate or advanced stages of the disease $(1,2)$. The early detection and diagnosis of liver cancer is an important issue that must be addressed. One study has shown that detection of liver cancer using magnetic resonance imaging (MRI) has a high degree of accuracy, but there are some limitations in the method in the identification of small lesions, particularly in patients with liver lesions smaller than $1 \mathrm{~cm}$, and in detecting liver cancer accompanied by liver cirrhosis (3). In order to detect small lesions more clearly, enhancement of the contrast agent in the contrast ratio and improvement of the MRI resolution of soft tissues are required. Gadolinium is effective for the imaging of the brain, kidneys and blood system, but is poor in imaging the hepatobiliary system. Therefore, it is necessary to investigate other contrast agents for imaging the liver using MRI. Superparamagnetic iron oxide (SPIO) has wide applications in the biomedical field and can be used not only as a drug-release carrier but also as a targeted probe carrier for monoclonal antibodies, polypeptides, hormones and genes (4-6). SPIO has recently garnered much attention in the field of molecular imaging research $(7,8)$. SPIO is a specific MRI contrast agent for liver imaging and plays an important role in the diagnosis of hepatocellular carcinoma, particularly small hepatocellular carcinoma $(9,10)$. In order to improve the targeting of SPIO and increase the efficiency of MRI in the diagnosis of hepatocellular carcinoma, modification of SPIO is necessary. In the present study, we synthesized SPIO using the chemical coprecipitation method. The nanoparticle SPIO-PEG-CTX was prepared using SPIO as the core, coating it with polyethylene glycol (PEG), and conjugating it with the targeting agent, chlorotoxin (CTX). This nanoparticle was studied using in vitro and in vivo MRI to determine its MR characteristics. 


\section{Materials and methods}

Statement of ethics. The present study was reviewed and approved by the Second Xiangya Hospital Institutional Review Board. All procedures involving animals were reviewed and approved by the Institutional Animal Care and Use Committee of Second Xiangya Hospital (SYXK 2012-003).

Reagents and animals. Reagents used to prepare the nanoparticle SPIO-PEG-CTX were as follows: iron trichloride hexahydrate (99\%), ferrous chloride tetrahydrate (99\%) (both from Aladdin Co.), PEG [average molecular weight (Mw), 6,000; Sinopharm Chemical Reagent Co. Ltd.] and CTX (98\%; Sigma-Aldrich).

Experimental animals included 8 New Zealand white rabbits of both genders weighing 2.0-3.0 kg provided by the Experimental Animal Center of Second Xiangya Hospital. One tumor-bearing rabbit was provided by Wuhan Union Hospital, Tongji Medical College, Huazhong University of Science and Technology.

MRI and SPIO hydrophobic nanoparticles. MRI was performed by Holland Philips Achieva 3.0T X Series superconducting MRI system and 16-channel SENSE XL Torso coil.

SPIO hydrophobic nanoparticles were prepared using the coprecipitation method. Iron trichloride hexahydrate $(1.081 \mathrm{~g}$, $4 \mathrm{mmol})$ and ferrous chloride $(0.397 \mathrm{~g}, 2 \mathrm{mmol})$ were mixed in a 250-ml 3-necked flask (Fig. 1). Then, $120 \mathrm{ml}$ deionized water was added into the flask. The flask was sealed, vacuumed of oxygen, and backfilled with nitrogen 3 times to create an anaerobic environment. Next, the whole iron solution system was stirred (mechanical agitation, faster than 1,500 rpm), and $0.2 \mathrm{ml}$ oleic acid was added to the mixed solution drop by drop. Then, $15 \mathrm{ml}$ of ammonium hydroxide (28\%) was added slowly to a 3-necked flask and the mixture was heated to $80^{\circ} \mathrm{C}$ in an ultrasonicator. The solution turned brown-black gradually during the addition of ammonium hydroxide. After $30 \mathrm{~min}$, the SPIO nanoparticles were prepared, which were dried by centrifugation, washed with anhydrous alcohol and vacuum dried at room temperature.

Surface modification of hydrophobic SPIO nanoparticles. Surface modification of hydrophobic SPIO nanoparticles was performed according to the methods of Veiseh et al (11) and Sun et al (12) as described below (Fig. 1). Step 1: PEG was added to a two-necked flask. One neck was connected to a water-separator, which was connected with a condenser tube, and the other neck was connected with a thermometer. Then, toluene was added to the flask to dissolve the PEG. The toluene/water mixture was removed by azeotroping at $110-130^{\circ} \mathrm{C}$ followed by cooling to room temperature to provide the product. Step 2: Succinic anhydride, the dried PEG6000 and 4-dimethylaminopyridine (DMAP) were stirred in methylene chloride for $24 \mathrm{~h}$. Then, the white solid was precipitated after treatment with ether. The solid was dissolved in isopropyl alcohol. The solid byproduct was removed, and carboxyl-terminated PEG was isolated following column chromatography and condensation. Step 3: PEG acid chloride was prepared by treatment of the carboxyl-terminated PEG with thionyl chloride under a nitrogen atmosphere for $1.5 \mathrm{~h}$. Step 4: Trifluoroethanol was added to the PEG acid chloride and stirred under nitrogen for $2 \mathrm{~h}$. Then, the mixture was heated to reflux for $3 \mathrm{~h}$, and the trifluoromethyl-functionalized PEG was purified using column chromatography. Step 5: Adenosine phosphosulfate (APS) and trifluoromethyl-functionalized PEG in a ratio of 1:2 were added to the solution made of PEG in dichloromethane. The solution was stirred overnight under nitrogen, and the PEG-modified silane was isolated following vacuum drying. Step 6: The PEG-modified silane was added to dry SPIO dispersed in cyclohexane at $50^{\circ} \mathrm{C}$ under ultrasound conditions to yield the hydrophilic SPIO modified by PEG-silane following removal of the cyclohexane. Step 7: An excess of ethanediamine was added to the SPIO modified by PEG-silane. After a 2-h reaction, the aminated SPIO was isolated by centrifugation following a wash with deionized water. Step 8: The carboxyl-activated iodoacetic acid was prepared by stirring iodoacetic acid, dicyclohexylcarbodiimide (DCC) and N-hydroxysuccinimide (NHS) in ethyl acetate for $3 \mathrm{~h}$. The carboxyl-activated iodoacetic acid dissolved in dimethyl sulfoxide (DMSO) was added to a suspension of SPIO in water. The reaction proceeded at room temperature overnight, and the ethyl iodoacetate-terminated SPIO was prepared following the removal of excess iodoacetic acid by filtration through a semipermeable membrane with a molecular weight limitation of 3,500. Step 9: Thioglycolic acid protected by acetylated sulfhydryl was prepared by reacting thioglycolic acid and acetyl chloride under anhydrous conditions. Carboxy-activated N-succinimidyl-S-acetylthioacetate (SATA) was prepared from thioglycolic acid, DCC and NHS at $0^{\circ} \mathrm{C}$. The carboxy-activated SATA was purified and then reacted with CTX $(\mathrm{pH}=8.5$ in bicarbonate buffer solution, $1 \mu \mathrm{g} / \mu \mathrm{l})$ for $4 \mathrm{~h}$ to yield SATA-CTX. Purification and concentration were performed by filtration through semipermeable membranes of a molecular weight range of 8,000-12,000. Step 10: Hydroxylamine hydrochloride and ethylenediaminetetraacetic acid (EDTA) was added to SATA-CTX, which reacted at room temperature for $1 \mathrm{~h}$. Then, the acetyl group of SATA-CTX was released to expose the reactive sulfhydryl group and the mercapto-modified CTX was formed. The mercapto-modified CTX was purified by filtration through semipermeable membranes with a molecular weight limitation of 3,500. The product reacted with ethyl iodoacetate-terminated SPIO by stirring in an ice-bath overnight to form the final product. The characterizations of the product were analyzed using infrared spectrometer and nuclear magnetic resonance spectrometer.

Establishment of the rabbit VX2 liver carcinoma model. Establishment of the rabbit VX2 liver carcinoma model was performed according to the methods of Chen et al (13), as described below (Fig. 2). The operation was carried out under sterile condition and anaesthesia with chloral hydrate. Tissue at the edge of the tumor was removed from the tumor-bearing rabbit (Fig. 2A). Necrotic tissue, fascia and other connective tissues were removed (Fig. 2B). The remained tissue was cut into small pieces (0.5-1 mm diameter) using scissors (Fig. 2C). A vertical incision was conducted below the xiphoid of healthy rabbits to expose the liver (Fig. 2D). The middle lobe of the liver was exposed using smooth forceps, and a $15-\mathrm{G}$ 




SATA

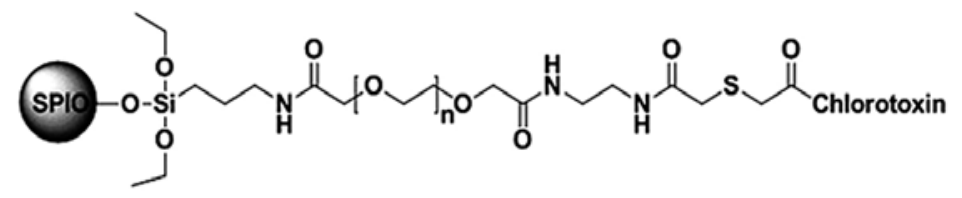

Figure 1. Surface modification of the hydrophobic SPIO nanoparticles.

needle was inserted sideways and upward into the visceral surface of the liver lobe and then $0.5-\mathrm{ml}$ tumor fragments were injected with a $5 \mathrm{ml}$ syringe. During this process, we ensured that the tumor fragments were injected into the liver parenchyma (Fig. 2E). Finally, the puncture site was covered with gelfoam after removal of the needle, and the incision was sutured after confirming no obvious bleeding (Fig. 2F).

MRI of SPIO and SPIO-PEG-CTX in vitro and in vivo. The MRI of SPIO and SPIO-PEG-CTX in vitro and in vivo were performed as follows. All scans were performed on an MR scanner (Philips Achieva 3.0T X Series; Phillips Healthcare, The Netherlands), using a 16-element phased array of SENSE XL Torso coil. The imaging protocol included the following: axial planes sense turbo spin-echo T2-weighted MRI [repetition time (TR)/echo time (TE), 1,565/70 millisecond (msec); NSA=3; flip angle, 90; matrix size, 252x192; field of view (FOV), 24x24 cm; section thickness: $4 \mathrm{~mm}$, gap $0.4 \mathrm{~mm}]$. Axial planes sense turbo spin-echo T1-weighted MRI (T1WI; TR/TE, 10/2.3 msec; NSA=3, flip angle, $15^{\circ}$; matrix, 252x193; FOV, 24x24 cm; section thickness: 4 mm; gap 0.4).
MRI scanning. MRI scanning was performed using the SPIO and SPIO-PEG-CTX solutions. The arrangements of the SPIO and SPIO-PEG-CTX solutions are shown in Fig. 3. The concentrations of the SPIO and SPIO-PEG-CTX stock solutions were both $10 \mathrm{mg} \mathrm{Fe} / \mathrm{ml}$. In samples no. 1-7, the solutions were prepared by adding 200, 100, 50, 40, 30, 20 and $10 \mu 1$ SPIO stock solution separately and diluting to $2 \mathrm{ml}$ in phosphate-buffered saline (PBS). PBS was used in sample no. 8. In the samples A-G the solutions were prepared by adding 200 , 100, 50, 40, 30, 20 and $10 \mu 1$ SPIO-PEG-CTX stock solution and diluting to $2 \mathrm{ml}$ with PBS. PBS was used in sample $\mathrm{H}$. The concentrations of samples no. 1-8 and A-H were: 1, 0.5, 0.25, $0.2,0.15,0.1,0.05$ and $0 \mathrm{mg} \mathrm{Fe} / \mathrm{ml}$.

MRI scanning of all 8 living rabbits was performed before and after the injection of the SPIO-PEG-CTX solution. Contrast enhanced T2 weighed images of the VX2 rabbits were acquired $30 \mathrm{~min}$ after intravenous bolus injection of $5 \mathrm{ml}$ SPIO-PEG-CTX at $25 \mu \mathrm{g} / \mathrm{ml}$.

The MR images were sent to a dedicated workstation and analyzed by 3 experienced radiologists. The region of interest (ROI) was $5 \times 5 \mathrm{~mm}$ for the SPIO and SPIO-PEG-CTX 

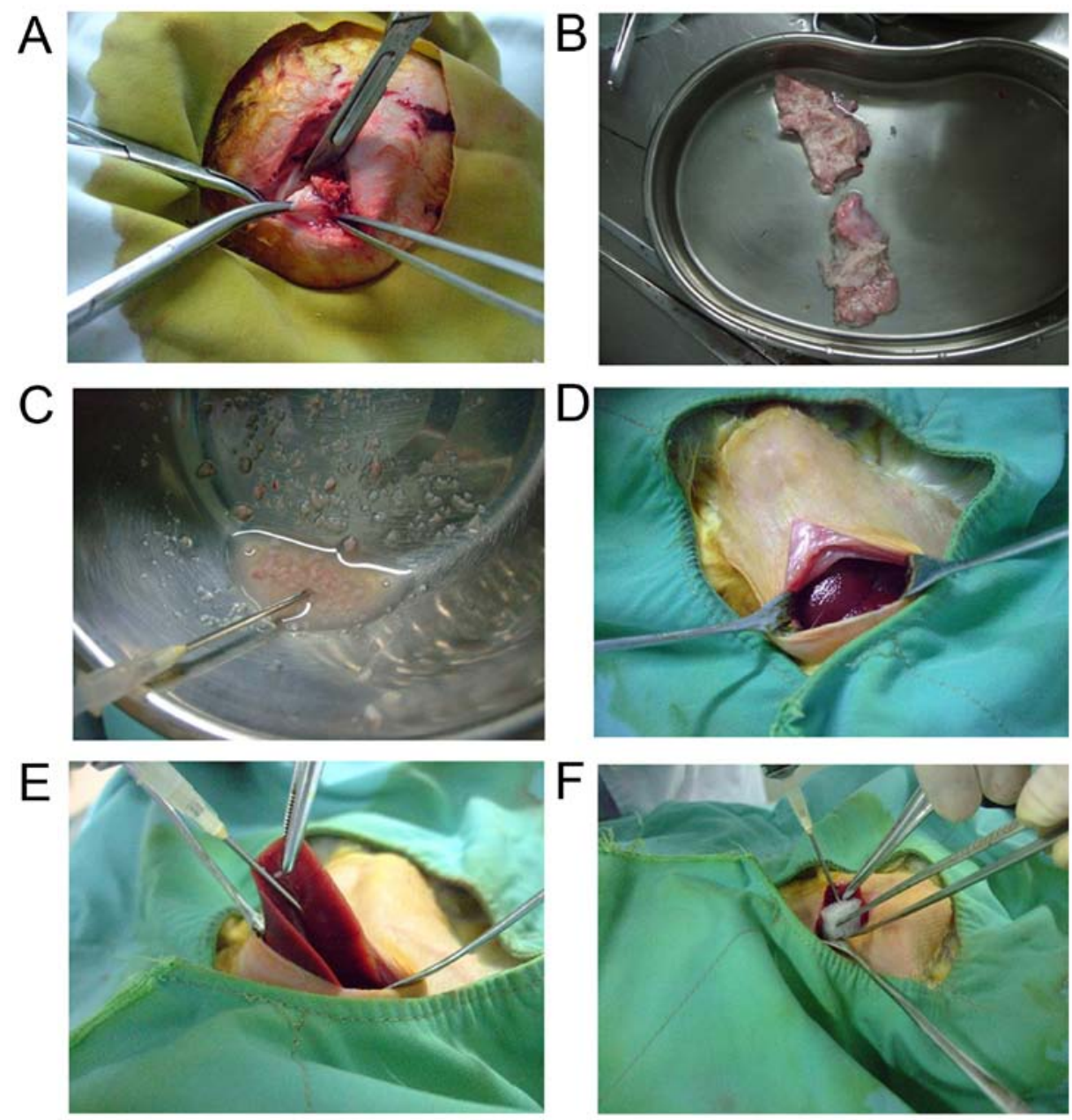

Figure 2. Process of establishing the rabbit VX2 hepatocarcinoma model. (A-F, steps are described in detail in the text).

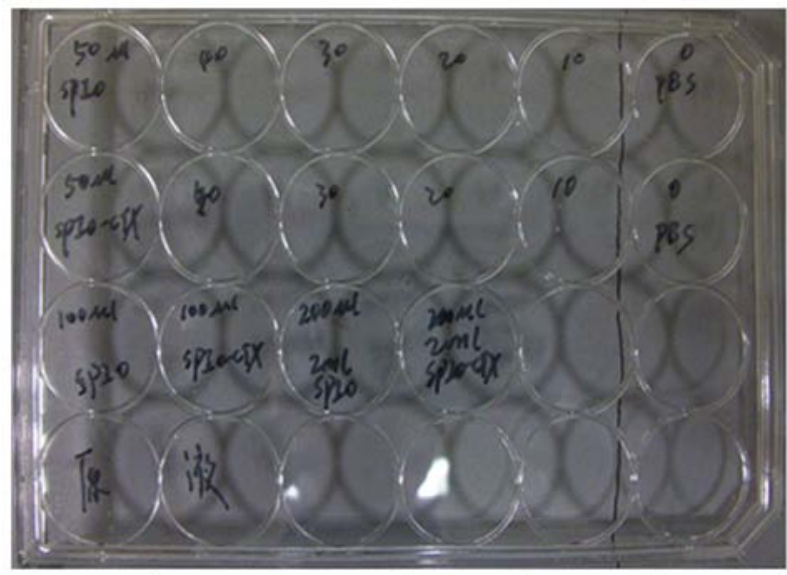

\begin{tabular}{|l|l|l|l|l|l|}
\hline (3) & (4) & (5) & (6) & (7) & (8) \\
\hline C & (D) & () & () & (C) & () \\
\hline (2) & (B) & (1) & (A) & 0 & 0 \\
\hline 0 & 0 & 0 & 0 & 0 & 0 \\
\hline
\end{tabular}

Figure 3. Arrangements of SPIO and SPIO-PEG-CTX solutions.

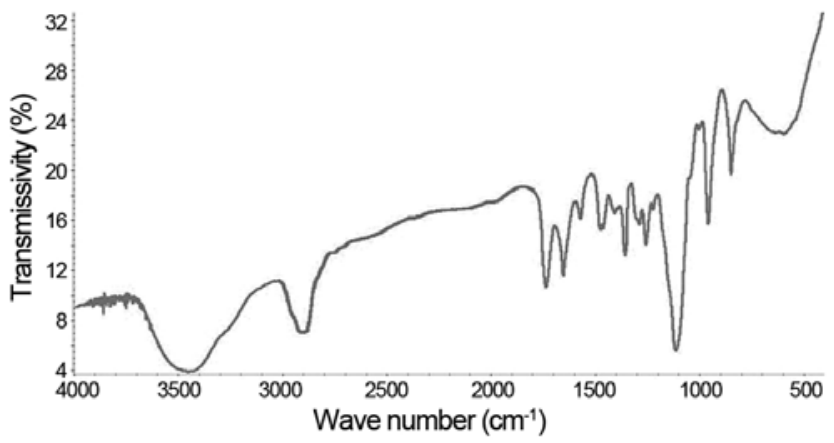

Figure 4. Infrared spectrum of the carboxyl-terminated PEG.

solution or rabbit liver tumor. Partial volume effect and pixels on the margin were not taken into account.

The liver tumors were removed and fixed in formalin after MRI scanning, and the sections were prepared for hematoxylin and eosin (H\&E) staining. The morphology and structure of the liver tumors were observed to determine the malignancy.

Data are presented as the mean \pm standard deviation and were analyzed using the paired t-test bySPSS 17.0 software. A difference with a P-value of $<0.05$ was considered to indicate a statistically significant result. 




Figure 5. Nuclear magnetic resonance spectroscopy of PEG-modified silane.

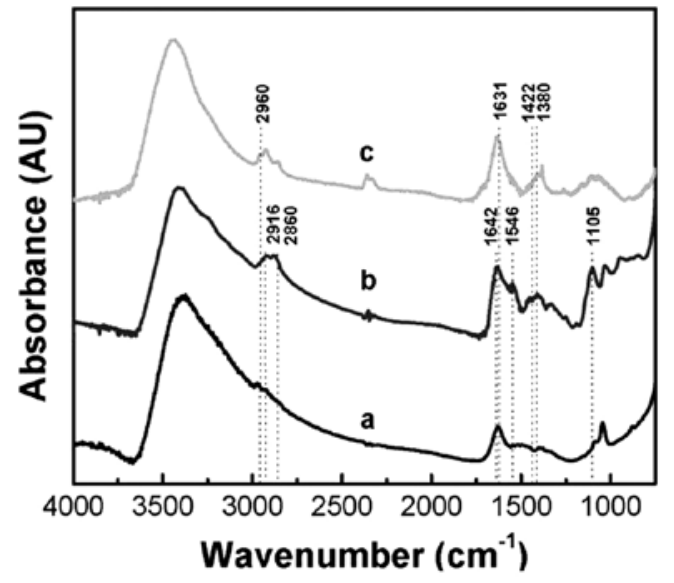

Figure 6.Infrared spectrums of $\mathrm{Fe}_{3} \mathrm{O}_{4}, \mathrm{Fe}_{3} \mathrm{O}_{4}$-PEG- $\mathrm{NH}_{2}$ and $\mathrm{Fe}_{3} \mathrm{O}_{4}$-PEG-CTX.

\section{Results}

Characterizations of the product. The infrared spectrum of the carboxyl-terminated PEG is shown in Fig. 4. The $3,000 \mathrm{~cm}^{-1}$ broad peak and $1,700 \mathrm{~cm}^{-1}$ double peak verified the success of the carboxyl modification. Nuclear magnetic resonance spectroscopy of PEG-modified silane is shown in Fig. 5. The infrared spectrums of $\mathrm{Fe}_{3} \mathrm{O}_{4}, \mathrm{Fe}_{3} \mathrm{O}_{4}$-PEG-NH $\mathrm{NH}_{2}$ and $\mathrm{Fe}_{3} \mathrm{O}_{4}$-PEG-CTX are shown in Fig. 6.

The MRI results of SPIO and SPIO-PEG-CTX in vitro. The MRI signal values of the SPIO and SPIO-PEG-CTX solutions are shown in Table I. The calibration curves of the concentrations and MRI signal values of the SPIO and SPIO-PEG-CTX solutions are shown in Fig. 7. There was a linear relationship between the concentrations and MRI values of the SPIO and SPIO-PEG-CTX solutions. With increasing SPIO and SPIOPEG-CTX concentrations, the T1WI value increased, and the T2WI values gradually decreased. The MRI value variations of the two solutions were basically identical. The MR images of the SPIO and SPIO-PEG-CTX solutions are shown in Fig. 8.

The MRI results of SPIO-PEG-CTX in vivo. Thirteen hepatic tumors were dissected from 8 rabbits. The MRI values of the tumors and the adjacent normal hepatic tissues on T2WI are shown in Table II. $S_{\text {Tumor- } 0}$ and $S_{\text {Normal-0, }}$, respectively, indicate the T2 signal value of the hepatic tumor and the normal adjacent hepatic tissue before injection of the SPIO-PEG-CTX solution. $S_{\text {Tumor-1 }}$ and $S_{\text {Normal-1 }}$, respectively, indicate the T2 signal value of the hepatic tumor and the adjacent normal hepatic tissue after injection of the SPIO-PEG-CTX solution. The data in Table II were analyzed by paired samples t-test using SPSS 17.0 software to evaluate differences between $S_{\text {Tumor- } 0}$ and $S_{\text {Tumor- } 1}, S_{\text {Normal- } 0}$ and $S_{\text {Normal } 1 .} . A \quad P<0.05$ was considered to indicate a significant difference. The $\mathrm{T} 2$ signal values of VX2 liver tumors and adjacent normal hepatic tissue before and after injection of the SPIO-PEG-CTX solution are shown in Table III. The MR images of a rabbit are shown in Fig. 9. Fig. 9A is the T1WI image. Fig. 9B is the plan-scanning T2WI image. Fig. $9 \mathrm{C}$ is the enhanced-scanning T2WI image. The VX2 liver tumor is indicated by an arrow. The tumor demonstrated hypointensity on plan-scanning T2WI, hyperintensity on T2WI, and the T2 signal intensity increased on enhancedscanning T2WI.

The mean $\mathrm{T} 2$ value of the $13 \mathrm{VX} 2$ tumors from 8 rabbits before injection of the SPIO-PEG-CTX solution 
Table I. MRI signal values of the SPIO and SPIO-PEG-CTX solutions.

\begin{tabular}{lcccccrrr}
\hline No. of holes & $1 / \mathrm{A}$ & 2/B & \multicolumn{1}{c}{$3 / \mathrm{C}$} & $4 / \mathrm{D}$ & $5 / \mathrm{E}$ & $6 / \mathrm{F}$ & $7 / \mathrm{G}$ & $8 / \mathrm{H}$ \\
\hline $\begin{array}{l}\text { Concentration of SPIO-PEG-CTX } \\
\text { and SPIO (mg Fe/ml) }\end{array}$ & 1 & 0.5 & 0.25 & 0.2 & 0.15 & 0.1 & 0.05 & 0 \\
T1WI value of SPIO & 23,012 & 16,484 & 8,876 & 6,572 & 4,539 & 3,210 & 2,055 & 1,105 \\
T1WI value of SPIO-PEG-CTX & 22,800 & 17,896 & 9,138 & 6,256 & 4,398 & 3,299 & 2,199 & 1,030 \\
T2WI value of SPIO & 5,124 & 19,840 & 37,550 & 41,368 & 43,877 & 46,734 & 49,372 & 52,370 \\
T2WI value of SPIO-PEG-CTX & 5,967 & 19,034 & 36,898 & 42,054 & 43,976 & 45,987 & 50,134 & 53,098 \\
\hline
\end{tabular}

Note: (As indicated in Fig. 3); in holes no. 1-7, the solutions were SPIO. In holes A-G the solutions were SPIO-PEG-CTX. MRI, magnetic resonance imaging; SPIO, superparamagnetic iron oxide; PEG, polyethylene glycol; CTX, chlorotoxin.


Figure 7. Calibration curves of the concentrations and MRI signal values.
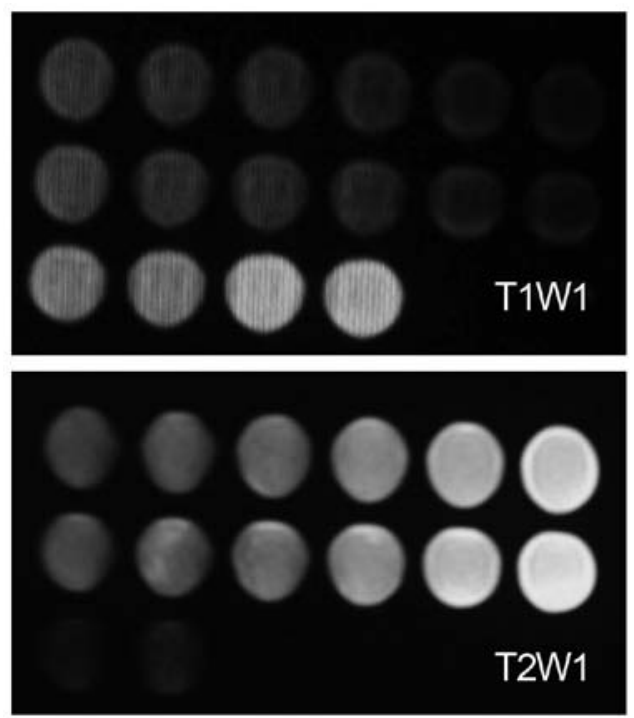

Figure 8. The MR images of SPIO and SPIO-PEG-CTX solution.

was $1,346 \pm 300.5$, and the mean value after injection was $1,161 \pm 331.5$. Thus, the enhanced-scanning $\mathrm{T} 2$ value was smaller than the plan-scanning $(\mathrm{P}=0.004<0.05)$. The mean $\mathrm{T} 2$
Table II. The MRI values of tumors and adjacent normal hepatic tissue on T2WI.

\begin{tabular}{rrrll}
\hline No. & $\mathrm{S}_{\text {Tumor- } 0}$ & $\mathrm{~S}_{\text {Tumor- } 1}$ & $\mathrm{~S}_{\text {Normal- } 0}$ & $\mathrm{~S}_{\text {Normal- }}$ \\
\hline 1 & 1,226 & $1,096.3$ & 585.9 & 441.1 \\
2 & 1,231 & $1,012.7$ & 580.7 & 394.3 \\
3 & 1,221 & 964.1 & 591.2 & 333.3 \\
4 & 951.2 & $1,025.4$ & 584.3 & 396.2 \\
5 & 943.1 & 679.8 & 583.2 & 331.5 \\
6 & $1,367.9$ & $1,083.1$ & 477.2 & 527.3 \\
7 & $1,363.4$ & $1,164.9$ & 474.9 & 772.4 \\
8 & $1,333.2$ & $1,195.8$ & 475.6 & 527.3 \\
9 & $1,335.7$ & $1,366.5$ & 473.1 & 770.1 \\
10 & $1,453.4$ & $1,427.3$ & 650.3 & 460.7 \\
11 & $1,449.7$ & $1,305.2$ & 645.9 & 407 \\
12 & $1,450.9$ & 771.7 & 648.5 & 237.1 \\
13 & $2,175.3$ & $2,004.3$ & 552.9 & 650.1 \\
\hline
\end{tabular}

MRI, magnetic resonance imaging.

values of the normal hepatic tissues near the 13 tumors before and after injection of SPIO-PEG-CTX were 563.4 \pm 67.8 and 

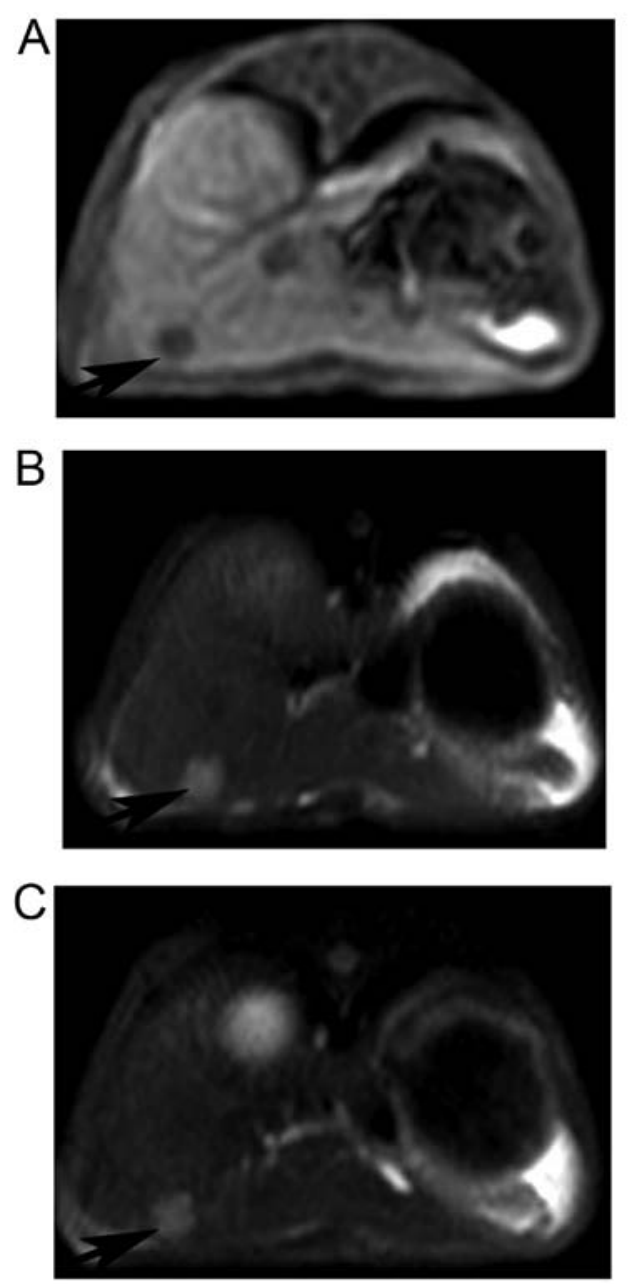

Figure 9. MRI of SPIO-PEG-CTX in vivo. (A-C are described in detail in the text).

Table III. The T2 signal values of VX2 liver tumors and adjacent normal hepatic tissue before and after injection of SPIO-PEG-CTX solution.

\begin{tabular}{lccc}
\hline & \multicolumn{1}{c}{$\mathrm{S}_{0}$} & $\mathrm{~S}_{1}$ & P-value \\
\hline Tumors & $1,346 \pm 300.5$ & $1,161 \pm 331.5$ & 0.004 \\
Adjacent normal & $563.4 \pm 67.8$ & $480.6 \pm 165.1$ & 0.202 \\
hepatic tissues & & & \\
\hline
\end{tabular}

$\mathrm{S}_{0}$ and $\mathrm{S}_{1}$ respectively, indicate T2 signal values of the 8 rabbits before and after injection of SPIO-PEG-CTX solution. SPIO, superparamagnetic iron oxide; PEG, polyethylene glycol; CTX, chlorotoxin.

$480.6 \pm 165.1$, respectively $(\mathrm{P}=0.202>0.05)$. Thus the $\mathrm{T} 2$ value of the normal hepatic tissue had no obvious change after injection of SPIO-PEG-CTX.

The histological findings as shown in Fig. 10. Fig. 10A shows the MR image of VX2 liver carcinoma on T1WI before the injection of SPIO-PEG-CTX (indicated by an arrow). The carcinoma was in the front of the liver and demonstrated hypointensity. Fig. 10B shows general sample observation of
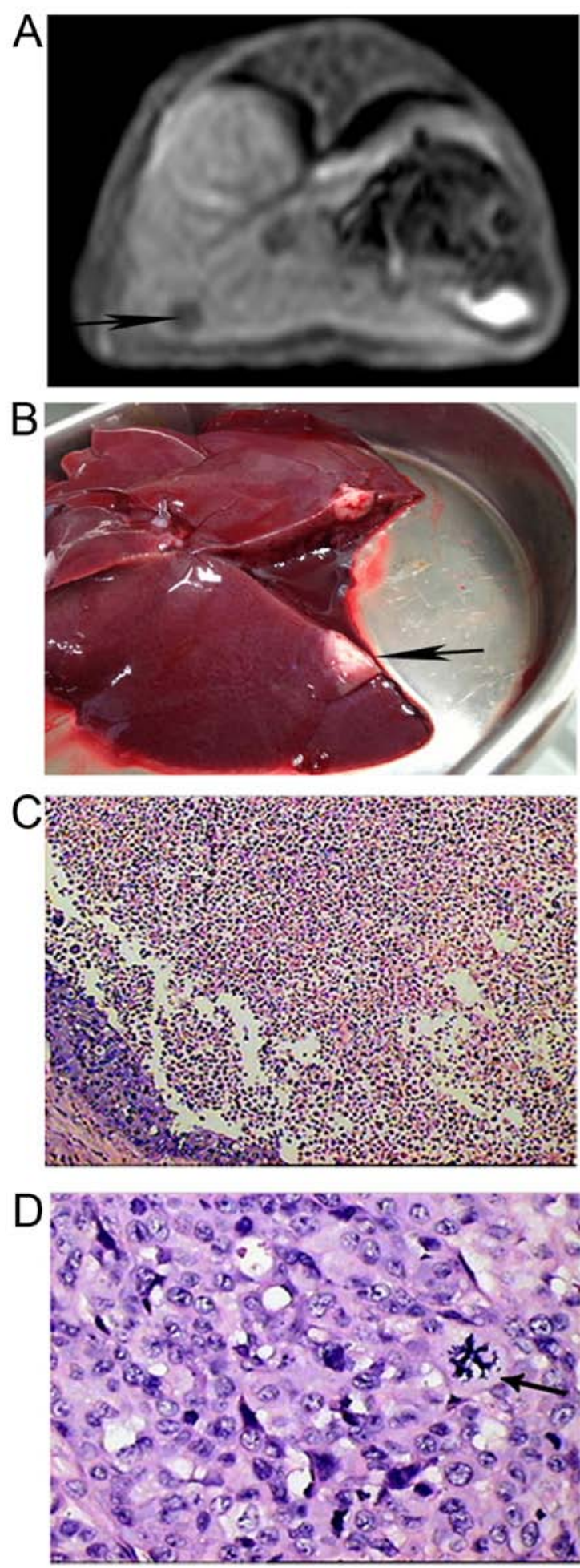

Figure 10. The histological findings of the rabbit VX2 hepatocarcinoma. (A-D are described in detail in the text).

the VX2 liver carcinoma (indicated by an arrow). There was a circumscribed whitish nodule at the edge of the liver. Fig. 10C is the pathological image of $\mathrm{H} \& \mathrm{E}$ staining at a magnification, x100; and Fig. 10D is the pathological image of H\&E staining at a magnification, $x 400$. The tumor cells were morphologically diverse, with obviously heterogeneous and trachychromatic nuclei; karyokinesis was also observed (indicated by an arrow in Fig. 10D). 


\section{Discussion}

Hepatocarcinoma is able to be detected by MRI in the clinical. However, there are some difficulties in the detection of small lesions, particularly in patients with liver lesions smaller than $1 \mathrm{~cm}$ or liver cancer accompanied by liver cirrhosis (3). The development of molecular imaging provides a new way for the early diagnosis of hepatocarcinoma. SPIO has a broad application in the biomedical field, and currently has become a hot spot in the field of molecular imaging research $(7,8)$.

Compared to gadolinium, SPIO can heighten the proton relaxation rate and shorten the $\mathrm{T} 2$ relaxation time. The reticuloendothelial system contains Kupffer cells, which can uptake SPIO and decrease the signal intensity of the liver. Since there are few or no Kupffer cells in the majority of malignant tumors of the liver, the signal intensity of malignant tumors remains unchanged when the liver takes up SPIO. In this case, the contrast in T2WI between the lesion and liver increases, which leads to negative enhancement (6).

The main ingredients of SPIO are $\mathrm{Fe}_{3} \mathrm{O}_{4}$ and some $\gamma-\mathrm{Fe}_{2} \mathrm{O}_{3}$ (14). $\mathrm{Fe}_{3} \mathrm{O}_{4}$ is black and strongly ferromagnetic, while $\gamma-\mathrm{Fe}_{2} \mathrm{O}_{3}$ is brown and has strong magnetic permeability. There are many methods by which to prepare magnetic nanoparticles, such as mechanical ball mill, coprecipitation, emulsification, sol-gel method, liquid-phase microwave dielectric heating, vapor deposition and hydrothermal method $(15,16)$. Chemical coprecipitation is the most common method, which is simple and inexpensive. Thus, stable and uniform SPIO was prepared using this method in the present study.

Previous studies have demonstrated that matrix metalloproteinase-2 (MMP-2) is expressed weakly in the liver tissues of chronic hepatitis and liver cirrhosis, but strongly in hepatocellular carcinoma $(17,18)$. The expression of MMP-2 is related to the differentiation, invasion, ability to metastasize and tendency of the tumor to recur in liver cancer (19). Thus, MMP-2 may be a new target that can be combined with a new molecular probe of hepatocarcinoma. CTX is a 36-amino acid peptide containing 1 tyrosine residue, 8 cysteine residues and 4 disulfide bonds. It is purified from the venom of the giant Israeli scorpion (Leiurus quinquestriatus). CTX can combine with MMP-2 selectively and specifically (20), which is strongly expressed in hepatocellular carcinomas. PEG is a cheap and commonly used surface-modification agent with good hydrophilicity and biocompatibility, but without immunogenicity and toxicity (21-24). It is soluble in water and many types of organic solvents and can be bound to nanoparticles by blocking or grafting. The nanoparticles modified with different functional groups display hydrophilicity and stability, can prolong the blood circulation time and can reduce phagocytosis. Therefore, we combined PEG as medium with SPIO and CTX to prepare SPIO-PEG-CTX nanoparticles. These nanoparticles can target hepatocellular carcinoma through the combination of CTX and MMP-2.

There are many dangling bonds on the surface of SPIO nanoparticles, which can be modified by targeted molecular probes with different functional groups such as carboxyl or amino groups or monoclonal antibodies. This type of modification can make magnetic nanoparticles reach the targeted organization and then amplify an imaging signal and improve image quality, effectively $(25,26)$. Surface modification is defined as changing the physicochemical properties of particles such as surface chemical structure, reaction characteristics, surface hydrophobicity, or surface chemical adsorption by physical or chemical methods. The unmodified nanoparticles have large specific surface area, surface hydrophobicity and static electricity. They can bring about precipitation and combine with plasma protein easily and then be cleared by the reticuloendothelial system in vivo, which may affect biomedical application of the particles. Therefore, SPIO was modified using ligand exchange in the present study. Whether the magnetic performance of SPIO could change after modification was investigated. In the present study, MRI signals of the SPIO and SPIO-PEG-CTX solutions had the same variations in vitro. These results suggest that SPIO-PEG-CTX can be used as a negative MRI contrast agent in T2WI sequence similar to SPIO.

The reticuloendothelial system contains Kupffer cells, which can uptake SPIO and decrease T2 signal intensity of the liver, while liver tumors do not uptake SPIO for the lack of Kupffer cells. In the present study, MR images of VX2 hepatocarcinoma rabbits injected with the SPIO-PEG-CTX (contrast) agent indicated an uptake of SPIO-CTX in the liver tumors which induced a decrease in the $\mathrm{T} 2$ signal intensity which was not observed in healthy regions of the liver. This suggests that CTX can alter the targeting of SPIO, and SPIOPEG-CTX was taken up by the liver tumors, but not by the normal liver tissue. Thus, the SPIO-PEG-CTX nanoparticles have potential for use in the early diagnosis of hepatocellular carcinoma. However, there were some limitations to the present study. The concentration of SPIO-PEG-CTX was not changed as an MR agent, and the time of enhanced-scanning was invariable. Thus, further investigation is warranted.

\section{Acknowledgements}

The present study was supported by the Natural Science Foundation of Hunan Province, China (no. 14JJ2034), the Natural Science Foundation of China (no. 81571784), the Foundation of Hunan Province and Technology Department, China (no. 2015SF2020-4), the Industry Research and Development Projects of the Development and Reform Commission of Hunan Province, China (no. 2060403-30499).

\section{References}

1. Kudo M: Hepatocellular carcinoma 2009 and beyond: From the surveillance to molecular targeted therapy. Oncology 75 (Suppl 1): S1-S12, 2008.

2. Jemal A, Bray F, Center MM, Ferlay J, Ward E and Forman D: Global cancer statistics. CA Cancer J Clin 61: 69-90, 2011.

3. Obuz F, Oksüzler M, Seçil M, Sağol O, Karademir S and Astarcioğlu H: Efficiency of MR imaging in the detection of malignant liver lesions. Diagn Interv Radiol 12: 17-21, 2006.

4. Bowman K and Leong KW: Chitosan nanoparticles for oral drug and gene delivery. Int J Nanomedicine 1: 117-128, 2006.

5. Cheng FY, Su CH, Yang YS, Yeh CS, Tsai CY, Wu CL, Wu MT and Shieh DB: Characterization of aqueous dispersions of $\mathrm{Fe}_{3} \mathrm{O}_{4}$ nanoparticles and their biomedical applications. Biomaterials 26 : 729-738, 2005.

6. Le Renard PE, Lortz R, Senatore C, Rapin JP, Buchegger F, Petri-Fink A, Hofmann H, Doelker E and Jordan O: Magnetic and in vitro heating properties of implants formed in situ from injectable formulations and containing superparamagnetic iron oxide nanoparticles (SPIONs) embedded in silica microparticles for magnetically induced local hyperthermia. J Magn Magn Mater 323: 1054-1063, 2011. 
7. Rosen JE, Chan L, Shieh DB and Gu FX: Iron oxide nanoparticles for targeted cancer imaging and diagnostics. Nanomedicine 8: 275-290, 2012.

8. Tassa C, Shaw SY and Weissleder R: Dextran-coated iron oxide nanoparticles: A versatile platform for targeted molecular imaging, molecular diagnostics, and therapy. Acc Chem Res 44: 842-852, 2011

9. Nishie A, Tajima T, Ishigami K, Ushijima Y, Okamoto D, Hirakawa M, Nishihara Y, Taketomi A, Hatakenaka M, Irie H, et al: Detection of hepatocellular carcinoma (HCC) using super paramagnetic iron oxide (SPIO)-enhanced MRI: Added value of diffusion-weighted imaging (DWI). J Magn Reson Imaging 31: 373-382, 2010.

10. Tanimoto A and Kuribayashi S: Application of superparamagnetic iron oxide to imaging of hepatocellular carcinoma. Eur J Radiol 58: 200-216, 2006.

11. Veiseh O, Sun C, Gunn J, Kohler N, Gabikian P, Lee D, Bhattarai N, Ellenbogen R, Sze R, Hallahan A, et al: Optical and MRI multifunctional nanoprobe for targeting gliomas. Nano Lett 5: 1003-1008, 2005.

12. Sun C, Veiseh O, Gunn J, Fang C, Hansen S, Lee D, Sze R, Ellenbogen RG, Olson J and Zhang M: In vivo MRI detection of gliomas by chlorotoxin-conjugated superparamagnetic nanoprobes. Small 4: 372-379, 2008.

13. Chen Z, Kang Z, Xiao EH, Tong M, Xiao YD and Li HB: Comparison of two different laparotomy methods for modeling rabbit VX2 hepatocarcinoma. World J Gastroenterol 21 : 4875-4882, 2015.

14. Gupta AK and Gupta M: Cytotoxicity suppression and cellular uptake enhancement of surface modified magnetic nanoparticles. Biomaterials 26: 1565-1573, 2005.

15. Laurent S, Forge D, Port M, Roch A, Robic C, Vander Elst L and Muller RN: Magnetic iron oxide nanoparticles: Synthesis, stabilization, vectorization, physicochemical characterizations, and biological applications. Chem Rev 108: 2064-2110, 2008

16. Hyeon T: Chemical synthesis of magnetic nanoparticles. Chem Commun 21: 927-934, 2003.

17. Lichtinghagen $\mathrm{R}$, Helmbrecht $\mathrm{T}$, Arndt $\mathrm{B}$ and Böker $\mathrm{KH}$ : Expression pattern of matrix metalloproteinases in human liver. Eur J Clin Chem Clin Biochem 33: 65-71, 1995.
18. Jacob A, Jing J, Lee J, Schedin P, Gilbert SM, Peden AA, Junutula JR and Prekeris R: Rab40b regulates trafficking of MMP2 and MMP9 during invadopodia formation and invasion of breast cancer cells. J Cell Sci 126: 4647-4658, 2013.

19. Wong JC, Chan SK, Schaeffer DF, Sagaert X, Lim HJ, Kennecke H, Owen DA, Suh KW, Kim YB and Tai IT: Absence of MMP2 expression correlates with poor clinical outcomes in rectal cancer, and is distinct from MMP1-related outcomes in colon cancer. Clin Cancer Res 17: 4167-4176, 2011.

20. Deshane J, Garner CC and Sontheimer H: Chlorotoxin inhibits glioma cell invasion via matrix metalloproteinase-2. J Biol Chem 278: 4135-4144, 2003.

21. Gryparis EC, Hatziapostolou M, Papadimitriou E and Avgoustakis K: Anticancer activity of cisplatin-loaded PLGA-mPEG nanoparticles on LNCaP prostate cancer cells. Eur J Pharm Biopharm 67: 1-8, 2007.

22. Gref R, Minamitake Y, Peracchia MT, Trubetskoy V, Torchilin V and Langer R: Biodegradable long-circulating polymeric nanospheres. Science 263: 1600-1603, 1994.

23. Moghimi SM and Szebeni J: Stealth liposomes and long circulating nanoparticles: Critical issues in pharmacokinetics, opsonization and protein-binding properties. Prog Lipid Res 42: 463-478, 2003.

24. Gref R, Lück M, Quellec P, Marchand M, Dellacherie E, Harnisch S, Blunk T and Müller RH: 'Stealth' corona-core nanoparticles surface modified by polyethylene glycol (PEG): Influences of the corona (PEG chain length and surface density) and of the core composition on phagocytic uptake and plasma protein adsorption. Colloids Surf B Biointerfaces 18: 301-313, 2000.

25. Juang JH, Wang JJ, Shen CR, Kuo CH, Chien YW, Kuo HY, Tsai ZT and Yen TC: Magnetic resonance imaging of transplanted mouse islets labeled with chitosan-coated superparamagnetic iron oxide nanoparticles. Transplant Proc 42: 2104-2108, 2010.

26. Ahmad T, Bae H, Rhee I, Chang Y, Lee J and Hong S: Particle size dependence of relaxivity for silica-coated iron oxide nanoparticles. Curr Appl Phys 12: 969-974, 2012. 